\title{
Peritoneal Panniculitis
}

National Cancer Institute

\section{Source}

National Cancer Institute. Peritoneal Panniculitis. NCI Thesaurus. Code C85004.

A disorder characterized by chronic inflammation and fibrosis of the adipose tissues in the peritoneal cavity. 